\title{
Direct Observation of Non-Markovian Radiation Dynamics in 3D Bulk Photonic Crystals
}

\author{
Ulrich Hoeppe, ${ }^{1,2}$ Christian Wolff, ${ }^{3}$ Jens Küchenmeister, ${ }^{3}$ Jens Niegemann, ${ }^{3}$ Malte Drescher, ${ }^{4}$ \\ Hartmut Benner, ${ }^{2}$ and Kurt Busch ${ }^{3}$ \\ ${ }^{1}$ Technische Hochschule Mittelhessen, Wilhelm-Leuschner-Straße 13, D-61169 Friedberg, Germany \\ ${ }^{2}$ Institut für Festkörperphysik, Technische Universität Darmstadt, Hochschulstraße 6 D-64289 Darmstadt, Germany \\ ${ }^{3}$ Institut für Theoretische Festkörperphysik and DFG-Center for Functional Nanostructures (CFN), \\ Karlsruhe Institute of Technology (KIT), Wolfgang-Gaede-Straße 1, D-76131 Karlsruhe, Germany \\ ${ }^{4}$ Department of Chemistry, University of Konstanz, D-78457 Konstanz, Germany
}

(Received 21 May 2011; published 26 January 2012)

\begin{abstract}
Since the very first proposition of photonic crystals, their influence on the dynamics of spontaneous emission has been of great interest. The radiation dynamics is described by an integration kernel whichin a spectral representation-comprises two equally important contributions: the Lamb shift and the radiative contribution to the linewidth. The latter is connected to the density of states via Fermi's golden rule. To our knowledge, we present the first spatially resolved measurement of the complete radiation dynamics in a photonic crystal and of its local density of states over a wide spectral range. To this end we study a single magnetic dipole situated in a photonic crystal with a band gap at microwave frequencies and find non-Markovian behavior in excellent agreement with $a b$ initio calculations.
\end{abstract}

DOI: $10.1103 /$ PhysRevLett.108.043603

PACS numbers: 42.50.Ct, 42.70.Qs, 76.50.+g

The history of photonic crystals (PhC) in the context of radiating emitters started in 1987 with Yablonovitch's seminal paper [1], where he proposed periodic dielectric structures as a means to inhibit spontaneous emission. Shortly thereafter, John and Wang found that even in an ideal $\mathrm{PhC}$ multiple scattering mediates a coherent interaction between the emitter and previously emitted radiation [2]. These memory effects eventually lead to nonMarkovian emission dynamics such as Rabi-like oscillations in the spectral vicinity of a photonic band edge [3]. This should be well distinguished from quantum emitters that are strongly coupled to spectrally localized modes such as cavity resonances [4] or disorder-induced resonances [5]. The latter systems are described by a Jaynes-Cummings-type Hamiltonian, whereas a PhC supports a continuum of photonic modes, and, therefore, our system is the implementation of a variant of the Dicke Hamiltonian [6].

There are mainly two popular experimental methods for investigating non-Markovian dynamics in Dicke-like systems in the optical regime [7]. In one class [8], pointlike emitters are deposited inside a photonic crystal and the fluorescence spectrum is observed. Another approach $[9,10]$ is to excite emitters (such as quantum dots) by a pump pulse and to observe the temporal decay of spontaneous emission into a narrow frequency band. In the former case, it is possible to measure both the linewidth and the Lamb shift, but one has to resort to emitters with a welldefined resonance frequency such as rare-earth atoms leading to poor spectral resolution. In the latter case, however, the observation frequency can be chosen arbitrarily at the expense of being unable to observe any shift in resonance. Finally, both methods record their data as functions of either time or frequency by using detectors outside the crystal. Hence, there is always the fundamental problem of separating the result of coherent feedback from ordinary filter effects by the bulk and the surface of the PhC [11]. While the former vanishes if the emitter is moved out of the crystal, the latter in principle remains as long as the detected radiation traverses the $\mathrm{PhC}$ and can mimic a nonexponential decay process. This can be overcome only by measuring the resonance with respect to some parameter other than frequency or time. One possibility is to use temperature-tuned quantum dots. However, the tuning range of such approaches is rather limited and systematic scans over appreciable frequency ranges are precluded. Instead, this may be employed for fine-tuning of emitters as has been done in 1D Dicke-like systems [12].

In our work, we use a small ferromagnetic sphere in a static magnetic field as a pointlike emitter. We place this single emitter at well-defined positions in a macroscopic three-dimensional $\mathrm{PhC}$. The external magnetic field allows us to tune the resonance frequency $\Omega$ of the collective excitations (magnons) of this spin system over a wide spectral range. In particular, this allows us to scan the resonance as a function of the magnetic field rather than the excitation frequency $\omega$. The resulting ferromagnetic resonance behaves like a classical dipole, so in a strict sense, we do not observe spontaneous emission. However, the theory to describe a radiating classical dipole is identical to the theory for spontaneous emission of an atom in the single excitation case [13]. Thus, the magnetic dipole behaves exactly like a single atom emitting one photon into an otherwise dark PhC. A detailed justification for modeling quantum optical problems with spin systems was given by Hahn [14]. 
It has been reported earlier [15] that the influence of a $\mathrm{PhC}$ on the radiation dynamics of an electric dipole is fully described by a time delay Green's function. We adapt the discussion in Ref. [15] to the case of magnetic coupling and obtain an analogous function $G(\tau)$ with $\tau=t-t^{\prime}$. Its Fourier transform is decomposed into real and imaginary parts $G(\omega)=\gamma(\omega)+i \lambda(\omega)$. In our formulation,

$$
\gamma(\omega)=\pi \beta \frac{N_{p}\left(\omega, \vec{r}_{0}\right)}{\omega}
$$

is half the linewidth and is directly connected to the local density of (magnetic) states

$$
N_{p}(\omega, \vec{r})=\int_{\mathrm{BZ}} d^{3} k \sum_{n} \delta\left(\omega-\omega_{n \vec{k}}\right)\left|\hat{\mu} \cdot \vec{H}_{n \vec{k}}(\vec{r})\right|^{2}
$$

at the location $\vec{r}_{0}$ of the dipole. Here, $\omega_{n \vec{k}}$ refers to the frequency of the propagating mode with wave vector $\vec{k}$ in the $n$th band. $\vec{H}_{n \vec{k}}(\vec{r})$ denotes the magnetic component of the corresponding field distribution, and the integration runs over the first Brillouin zone (BZ). Equation (2) has to be averaged over all relevant dipole orientations $\hat{\mu} ; \beta$ is an effective coupling strength and was determined by measuring the free-space linewidth of our emitter over the experimental frequency window. The imaginary part of the Green's function causes a resonance shift (the classical analogon to the optical Lamb shift) and is connected to $\gamma(\omega)$ via

$$
\lambda(\omega)=\frac{1}{\pi} \mathcal{P} \int_{0}^{\infty} d \omega^{\prime} \frac{\gamma\left(\omega^{\prime}\right)}{\omega-\omega^{\prime}} .
$$

Superlogarithmically divergent terms of this principal value integral have to be disregarded as they describe dipole self-interaction that is already contained in the free electron mass [15]. Some authors prefer to relate the linewidth to the imaginary part and the resonance shift to the real part. This can be easily accomplished by introducing a phase factor.

In a conventional fluorescence experiment, the detector would record the power spectrum as a function of $\omega$ :

$$
P_{\text {rec }}(\omega, \Omega)=P_{0} \frac{2 F(\omega) \gamma(\omega)}{[\gamma(\omega)]^{2}+[\omega-\Omega-\lambda(\omega)]^{2}} .
$$

All filter effects have been condensed in a filter function $F(\omega)$. It is crucial to understand the basic difference between $\omega$ and $\Omega$. The former is the frequency of a spectral component of the emitted radiation, whereas the latter is the bare resonance frequency of the radiating dipole. In our setup, $\Omega$ is approximately proportional to the external magnetic field $(\Omega / H \approx 2 \pi \times 2.81 \mathrm{MHz} / \mathrm{Oe})$, while $\omega$ corresponds to the microwave excitation frequency. Whenever the frequency-dependent variations of $\gamma(\omega)$ and $\lambda(\omega)$ occur on a scale comparable to the resonance linewidth, an $\omega$ scan of $P_{\text {rec }}(\omega, \Omega)$ will directly show a distortion of the line shape as indicated in Figs. 1(b) and 1(c).
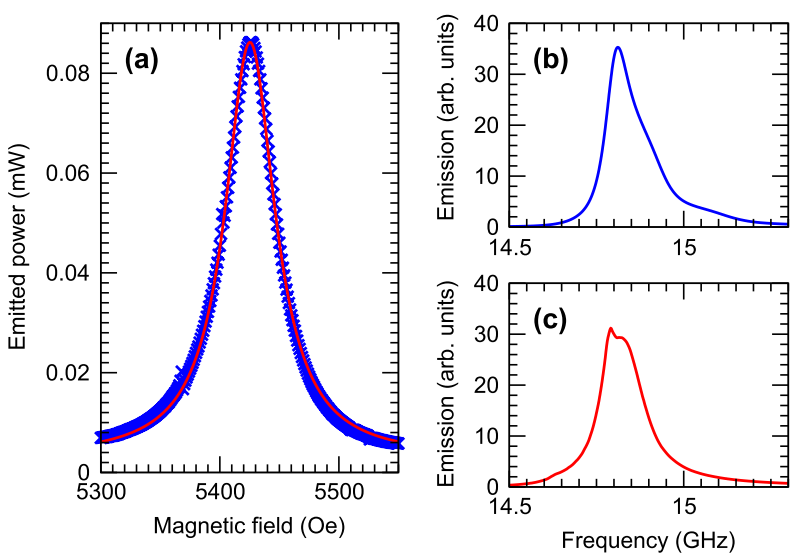

FIG. 1 (color online). Ferromagnetic resonance of the YIG sample at position 1. (a) Experimental scan with respect to the external magnetic field at fixed microwave frequency $\omega / 2 \pi=$ 14.9 GHz. The solid (red) curve is a Lorentzian fit to the data points (blue crosses). (b),(c) Frequency scans at a constant magnetic field which corresponds to a bare emitter frequency of $\Omega / 2 \pi=14.9 \mathrm{GHz}$. The resonance curves were reconstructed from linewidth and shift data obtained from experimental field scans at various values of $\omega$ (b) and from corresponding theoretical calculations (c).

Such a deviation from a Lorentzian is characteristic for non-Markovian dynamics and corresponds to nonexponential emission in the time domain. In contrast to that, by keeping $\omega$ fixed, Eq. (4) describes a perfect Lorentzian resonance as a function of $\Omega$. This means that the radiation dynamics for fixed excitation frequency $\omega$ can be characterized completely and without any systematic distortion by observing the emission as a function of $\Omega$, i.e., of the external magnetic field.

In the experiment we used a single crystal yttrium iron garnet (YIG) sphere with a diameter of $1.76 \mathrm{~mm}$. The intrinsic linewidth of this emitter is below 1 Oe, so nonradiative processes are weak compared to the effect of radiation damping, which leads to a free-space linewidth between 3 and 30 Oe in the observed frequency range of 8-17 GHz [16].

Our PhC was similar to the one investigated in Ref. [17]. It consisted of cylindric alumina rods (diameter $3.05 \pm$ $0.05 \mathrm{~mm}$, permittivity 9.8 according to the manufacturer's specifications) in a woodpile structure [18] with a rod spacing of $10.6 \pm 0.3 \mathrm{~mm}$ (cf. Fig. 2). The total dimensions of the $\mathrm{PhC}$ were $156 \times 156 \times 57 \mathrm{~mm}^{3}$, so it was possible to place it into the gap of a VARIAN V-3900 electromagnet, which was controlled by a Bruker BH15 interface. The geometry of the $\mathrm{PhC}$ allowed us to place the YIG sphere at different positions by means of a small plastic tube. We excited the ferromagnetic resonance with a small nearby loop antenna that was fed by an HP83752B microwave sweep generator. A horn antenna outside the crystal received the emitted radiation. We measured the resonance at a fixed frequency $\omega$ as a function of the magnetic field. A certain amount of direct 
coupling between the exciting loop antenna and the receiving horn antenna was inevitable, so we added a compensation network that eliminated this direct irradiation: Part of the generator signal was split off with a directional coupler, adjusted in amplitude and phase, and fed back to the output signal of the receiving horn antenna. The adjustment was done such that the cross talk between the input and the output antennae was completely compensated and the remaining signal solely resulted from the YIG sample. We determined the output power at the end of the microwave circuit by measuring the voltage at a detector diode using an HP3457A digital voltmeter. For each frequency setting, we first readjusted the compensation network such that the detected signal vanished with the YIG sample being off-resonance. Then, we recorded the resonance curve on a variation of the magnetic field around resonance. Finally, the resonance linewidth was determined by a Lorentzian fit to the measured data. The resonance shift was determined as the deviation of the center of this Lorentzian fit from the expected "bare resonance field" defined by the condition $\Omega=\omega$. Since the shift is a small quantity, we had to consider very carefully the dependence of $\Omega$ on $H$ including minor nonlinear corrections well known from the literature [19].

To verify the spectral properties of our PhC, we measured the transmission characteristics without any emitter inside (not shown). We compared it to theoretical spectra obtained with our in-house implementation of the Fourier modal method [20] and found agreement better than $2 \%$ with respect to the stop band frequencies. These results are consistent with corresponding band structure calculations using the software package MPB [21] at a spatial discretization of $32 \times 32 \times 32$ points per unit cell. According to these calculations, our $\mathrm{PhC}$ exhibits a complete photonic band gap between 13.0 and $14.3 \mathrm{GHz}$.

Next, we measured the emitter resonance inside the $\mathrm{PhC}$. Figure 1(a) shows a typical emission line for an excitation frequency of $14.9 \mathrm{GHz}$, i.e., exactly at the upper band edge. Here, in a frequency scan, strong line distortion would be expected. Our experimental field scan, however, shows an almost perfect Lorentzian (like all other lines observed with respect to $\Omega$ ). This demonstrates that by our experimental approach any filter effects have been systematically removed from the line shape, and the full radiation response is solely encoded in the linewidth and the exact resonance position. In Fig. 3, we depict the frequency dependence of the linewidth and the resonance shift for two well-defined emitter locations (cf. Fig. 2). In the band gap, spontaneous emission is strongly suppressed; the linewidth is reduced by an order of magnitude with respect to free space. Below and above the band gap, the linewidth considerably exceeds its free-space value. Superimposed we find a series of more or less pronounced peaks which we attribute to standing modes inside the PhC (Fabry-Pérot resonances due to the crystal's finite size). Above the band
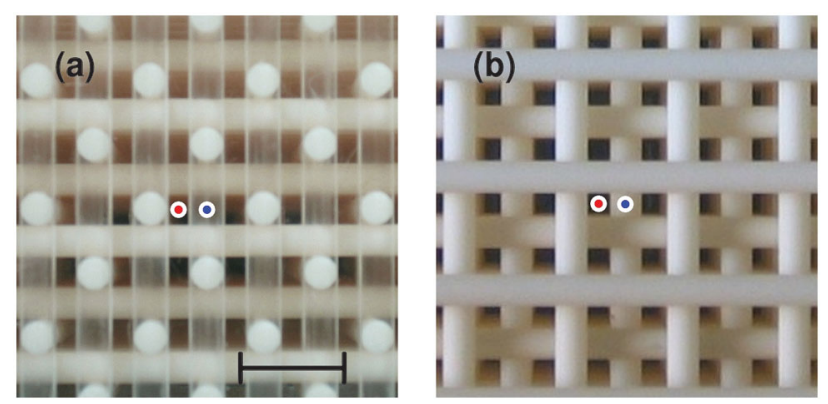

FIG. 2 (color). Side-view (a) and top-view (b) photographs of the experimental photonic crystal (the scale bar corresponds to $10 \mathrm{~mm}$ ). Experimental data were recorded for two different emitter positions: position 1 close to a dielectric rod, indicated by a red dot, and position " 2 " at a maximum distance from any dielectric rod, indicated by a blue dot. The dot size corresponds to the size of the YIG sphere; white rings were added for better contrast.

gap, we also find a strong dependence on the emitter position, which is related to the spatial distribution of the photonic modes. Here, the magnetic field of the Bloch modes is more strongly concentrated inside the dielectric rods and falls off outside. Consequently, the emitter couples more efficiently to the propagating modes if positioned next to a rod. Wherever the linewidth spectrum has a minimum, the resonance shift exhibits steep negative slopes. In optics, this effect is usually referred to as the anomalous Lamb shift. It occurs most clearly across the band gap, but also-at position "1"-for frequencies above the gap where the linewidth fluctuates due to finite-size effects.

We also compare these curves with theoretical results obtained from $a b$ initio local density of states calculations which were based on the technique described in Ref. [22], using approximately $2.4 \times 10^{6}$ tetrahedra with proper handling of the crystal's symmetries [23]. The principal value integral [Eq. (3)] was evaluated semianalytically for a piecewise linear interpolation of the numerical local density of states on the frequency window $0-30 \mathrm{GHz}$ with a sampling of $2.8 \mathrm{MHz}$. Overall, we find very good agreement. There are three main sources of deviation. First, the theoretical curves are systematically offset in frequency by approximately $200 \mathrm{MHz}$. This suggests that the dielectric constant of the alumina rods slightly differs from the vendor's specification. Second, our simulations do not show the finite-size oscillations observed in the experiment, since we considered an infinitely extended PhC. Third, the theoretical resonance shift is subject to an additional offset due to the finiteness of the numerical integration domain. This offset scales logarithmically with both the emitter frequency and the integration cutoff. Therefore, it is very smooth and does not affect the local spectral features.

Finally, we reconstructed the emission spectrum by evaluating Eq. (4) for a fixed emitter resonance $\Omega$ (i.e., for a constant magnetic field) using the previously obtained 

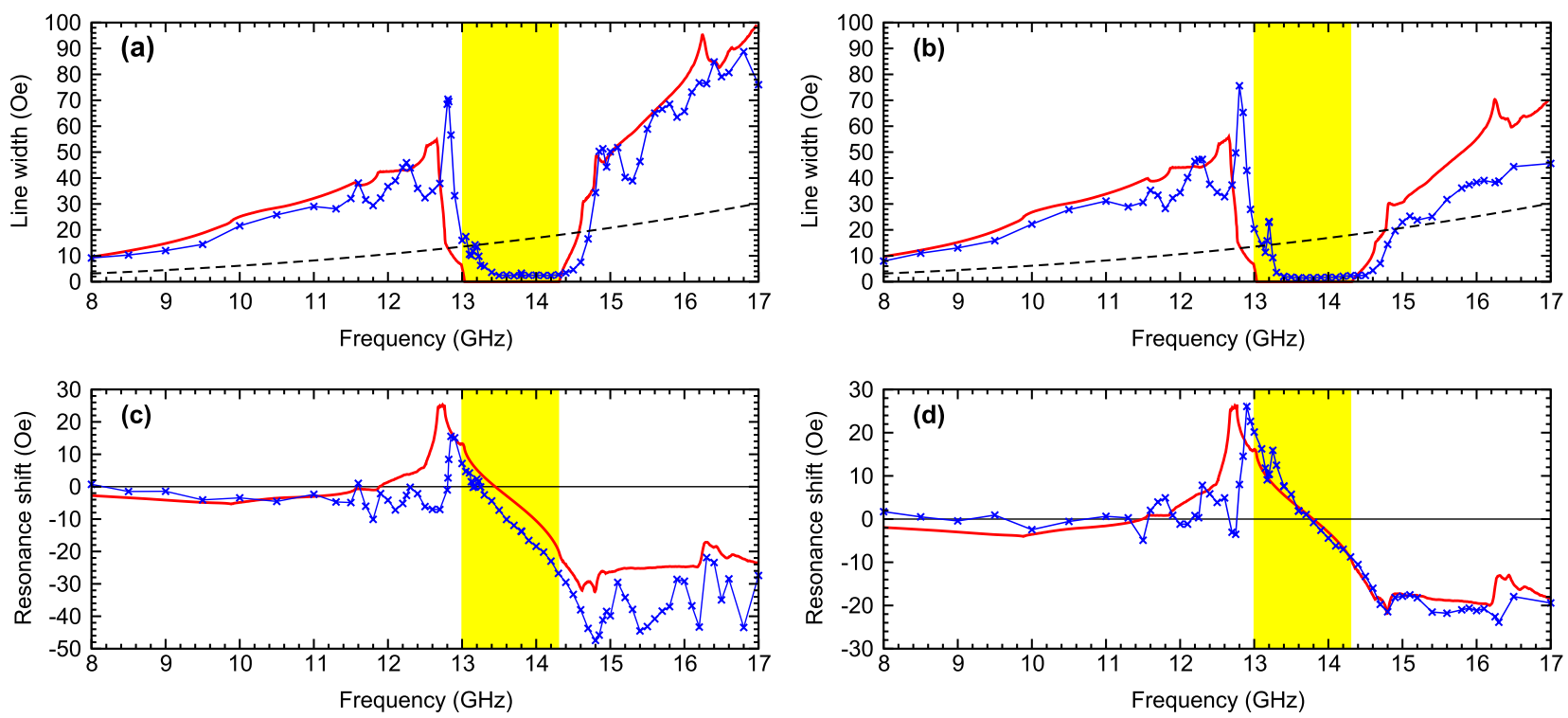

FIG. 3 (color online). Linewidth and resonance shift obtained at the emitter position 1 [(a) and (c), respectively] and position 2 [(b) and (d)]. Experimental data points are depicted as blue crosses. The connecting blue solid lines were added to guide the eye. The results of corresponding ab initio numerics are shown as red solid lines. The computed photonic band gap is indicated by yellowshaded areas. For comparison, the vacuum linewidth based on our experimentally determined coupling constant $\beta$ is indicated by a dashed line.

theoretical and experimental linewidth and shift data (cf. Fig. 3), converting them to frequency units, and setting the filter function $F(\omega)$ to unity. The resulting spectra with respect to $\omega$ [depicted in Figs. 1(b) and 1(c)] represent the emission at the position of the emitter and are free of filter effects. In vacuum, these curves would be Lorentzian, whereas the nearby band gap causes a serious deformation with a long tail towards higher frequencies. The qualitative agreement between both curves is very good, apart from minor deviations resulting from the local spectral features of $\gamma(\omega)$ and $\lambda(\omega)$ around $14.9 \mathrm{GHz}$. These clearly nonLorentzian emission lines are a direct evidence for a nonMarkovian radiation process.

In conclusion, we have fully characterized the linear radiation dynamics of a single emitter situated at different well-defined positions within a woodpile $\mathrm{PhC}$ and experimentally probed the local density of magnetic states by means of ferromagnetic resonance. The experiment covers a spectral range of more than one octave, and the results are in excellent agreement with theory and numerical predictions regarding linewidth and anomalous resonance shift. We would like to stress that the coupling constant $\beta$ was determined from free-space measurements, so that there were no adjustable parameters in the $\mathrm{PhC}$ experiment. The quantitative agreement between our experimental and theoretical time delay Green's function, which is the central quantity in the context of emission from active materials, establishes a firm basis for the physics of modified radiation dynamics in photonic crystals. Our new experimental method can be directly adapted to study the photonic properties of other structured media such as metamaterials or disordered media.

We thank Elmar Dormann and Lasha Tkeshelashvili for stimulating and fruitful discussions.

[1] E. Yablonovitch, Phys. Rev. Lett. 58, 2059 (1987).

[2] S. John and J. Wang, Phys. Rev. Lett. 64, 2418 (1990).

[3] S. John and T. Quang, Phys. Rev. A 50, 1764 (1994).

[4] T. Yoshie et al., Nature (London) 432, 200 (2004).

[5] L. Sapienza, H. Thyrrestrup, S. Stobbe, P. Garcia, S. Smolka, and P. Lodahl, Science 327, 1352 (2010).

[6] J. T. Shen and S. Fan, Opt. Lett. 30, 2001 (2005).

[7] S. Noda, M. Fujita, and T. Asano, Nature Photon. 1, 449 (2007).

[8] L. Qiong, S. Hongwei, W. Wei, B. Xue, W. Yu, D. Biao, X. Lin, and H. Wei, Opt. Lett. 35, 2898 (2010).

[9] I. S. Nikolaev, P. Lodahl, A. F. van Driel, A. F. Koenderink, and W. L. Vos, Phys. Rev. B 75, 115302 (2007).

[10] P. Lodahl, A. F. van Driel, I. S. Nikolaev, A. Irman, K. Overgaag, D. Vanmaekelbergh, and W. L. Vos, Nature (London) 430, 654 (2004).

[11] C. Schuler, C. Wolff, K. Busch, and M. Florescu, Appl. Phys. Lett. 95, 241103 (2009).

[12] H. Thyrrestrup, L. Sapienza, and P. Lodahl, Appl. Phys. Lett. 96, 231106 (2010).

[13] K. Busch, N. Vats, S. John, and B. C. Sanders, Phys. Rev. E 62, 4251 (2000).

[14] E. L. Hahn, Concepts Magn. Reson. 9, 69 (1997).

[15] N. Vats, S. John, and K. Busch, Phys. Rev. A 65, 043808 (2002). 
[16] R. W. Sanders, D. Paquette, V. Jaccarino, and S.M. Rezende, Phys. Rev. B 10, 132 (1974).

[17] E. Özbay, A. Abeyta, G. Tuttle, M. Tringides, R. Biswas, C. T. Chan, C. M. Soukoulis, and K. M. Ho, Phys. Rev. B 50, 1945 (1994).

[18] K. Ho, C. Chan, C. M. Soukoulis, R. Biswas, and M. Sigalas, Solid State Commun. 89, 413 (1994).
[19] J. E. Mercereau, J. Appl. Phys. 30, S184 (1959).

[20] S. Essig and K. Busch, Opt. Express 18, 23258 (2010).

[21] S. Johnson and J. Joannopoulos, Opt. Express 8, 173 (2001).

[22] K. Busch and S. John, Phys. Rev. E 58, 3896 (1998).

[23] I. S. Nikolaev, W. L. Vos, and A. F. Koenderink, J. Opt. Soc. Am. B 26, 987 (2009). 